KJeldgaArd, N. O., Maalge, O. \& Schaechter, M. (1958). J. gen. Microbiol. 19 607-616

\title{
The Transition Between Different Physiological States During Balanced Growth of Salmonella typhimurium
}

\author{
By N. O. KJELDGAARD, O. MAALøE AND M. SCHAECHTER*
}

State Serum Institute, Copenhagen, Denmark

SUMMARY: When cultures of Salmonella typhimurium undergoing balanced growth are shifted from one medium to another, a definite pattern of rate changes is observed. Shifts from a low to a high growth rate result in a strict succession of events: RNA synthesis is immediately affected and its rate rapidly increases to that characteristic of the new medium; the increase in optical density shows a lag of a few minutes before the new rate is attained; DNA synthesis and cell division, on the other hand, continue at the old rate for appreciable periods of time and then abruptly shift to the new rates. The times at which these shifts take place are, at $37^{\circ}$, invariably 20 and 70 min., regardless of the actual growth rates before and after the shift. This rate maintenance effect on DNA synthesis and cell division is discussed in terms of specific rate-controlling mechanisms.

In the preceding paper (Schaechter, Maaløe \& Kjeldgaard, 1959) we showed that the size and chemical composition of Salmonella typhimurium vary exponentially with the growth rate afforded by different media. The present paper describes the transition from one state of balanced growth to another. The terms 'balanced growth' and 'cell' as used by us, have been defined in the preceding paper. Two types of experiments were performed, both involving precisely timed changes of medium at constant temperatures; one going to a higher growth rate (shift $u p$ ), and the other to a lower rate (shift down).

The shift up, exemplified by going from a glucose salt medium to nutrient broth, is characterized by an orderly dissociation of the main synthetic activities. Upon addition of broth, the rates of synthesis of ribonucleic acid (RNA) and of total mass immediately increase, whereas deoxyribonucleic acid (DNA) synthesis and cell division continue at the old rate for considerable periods of time. The new state of balanced growth is reached when, after about $70 \mathrm{~min}$. (at $37^{\circ}$ ), the rate of cell division abruptly changes from the preshift value to the definitive broth rate. We interpret this 'rate maintenance effect' as evidence for the existence of separate rate-controlling mechanisms.

The shift down is rapidly effected by filtration of, say, a nutrient broth culture and resuspension of the cells in simple defined medium. It is characterized by a period during which cell division and DNA synthesis continue in the absence of net synthesis of RNA and total mass. By the time RNA and mass begin to increase the cells have already approached the size and the composition typical of balanced growth in the simpler medium. This behaviour

\footnotetext{
* Present address: Department of Microbiology, College of Medicine, University of
} Florida, Gainesville, Florida, U.S.A. 
demonstrates that certain synthetic abilities are repressed during growth in a rich medium and that it takes considerable time to re-establish all the functions necessary for growth in the poorer medium.

\section{METHODS}

Strains. Salmonella typhimurium, see Schaechter etal. (1958). Escherichia coli, strain K 12 (58-161), methionine-requiring, was obtained from Dr W. Hayes. E. coli strain ML 37, an inducible $\beta$-galactosidase former, was obtained from Dr J. Monod.

Media. See Schaechter et al. (1958).

\section{General procedures}

Shift up: to a culture grown for several generations under conditions of balanced growth was added an equal volume of double-strength medium supporting faster growth.

Shift down: a culture maintained under conditions of balanced growth for several generations was rapidly filtered on a membrane filter (MembranfilterGesellschaft, Sartorius-Werke A. G., Göttingen). The filter was washed with portions of the new medium, the bacteria shaken off and resuspended. This operation usually took 2-3 min. All fresh media were pre-warmed to $37^{\circ}$.

Conditions of aeration, bacterial density, chemical analysis, nuclear staining, colony counts, etc., were described in Schaechter et al. (1958).

\section{RESULTS}

A typical shift $u p$ at $37^{\circ}$ between minimal medium with glucose as energy source (average generation time: $\mathbf{5 0} \mathrm{min}$.) and nutrient broth (average generation time: 22 min.) is illustrated in Fig. 1 for Salmonella typhimurium. After addition of broth a characteristic dissociation of the main synthetic activities was observed, as follows. (1) RNA synthesis was immediately and strongly stimulated and, for a short period, took place at a rate higher than that characteristic for broth. (2) Mass synthesis (as inferred from optical density measurements) rapidly increased and, after about $5 \mathrm{~min}$., reached the rate typical of balanced growth in broth. (3) DNA synthesis remained unaffected for about 20 min., at which time its rate suddenly changed from the pre-shift value to the definitive broth value. (4) The average number of nuclei/cell stayed constant at about 1.5 until about $35 \mathrm{~min}$. after the shift, and then, within 15-20 min., increased to 3.0. (5) Cell division, finally, continued at the pre-shift rate for about $70 \mathrm{~min}$., when the rate suddenly increased and assumed the definitive broth value. The cells now reached the size and composition previously established for balanced growth of this organism in broth (see Schaechter et al. 1958).

The transition pattern illustrated in Fig. 1 has three distinguishing features: first, the order in which the synthetic activities respond to the addition of broth; secondly, the precise maintenance of the pre-shift rates of DNA synthesis and cell division for about 20 and $70 \mathrm{~min}$., respectively; and thirdly, 


\section{Transition between states of balanced growth}

the abruptness with which, at these times, the definitive broth rates are established.

These characteristics are reproduced with remarkable constancy with related organisms. In a glucose salt medium (with methionine added) and in broth Escherichia coli, strain K 12 (58-161), exhibited growth rates similar to those obtained with Salmonella typhimurium, and the transition pattern of Fig. I was almost duplicated. Strain ML37 of $E$. coli was tested in a shift

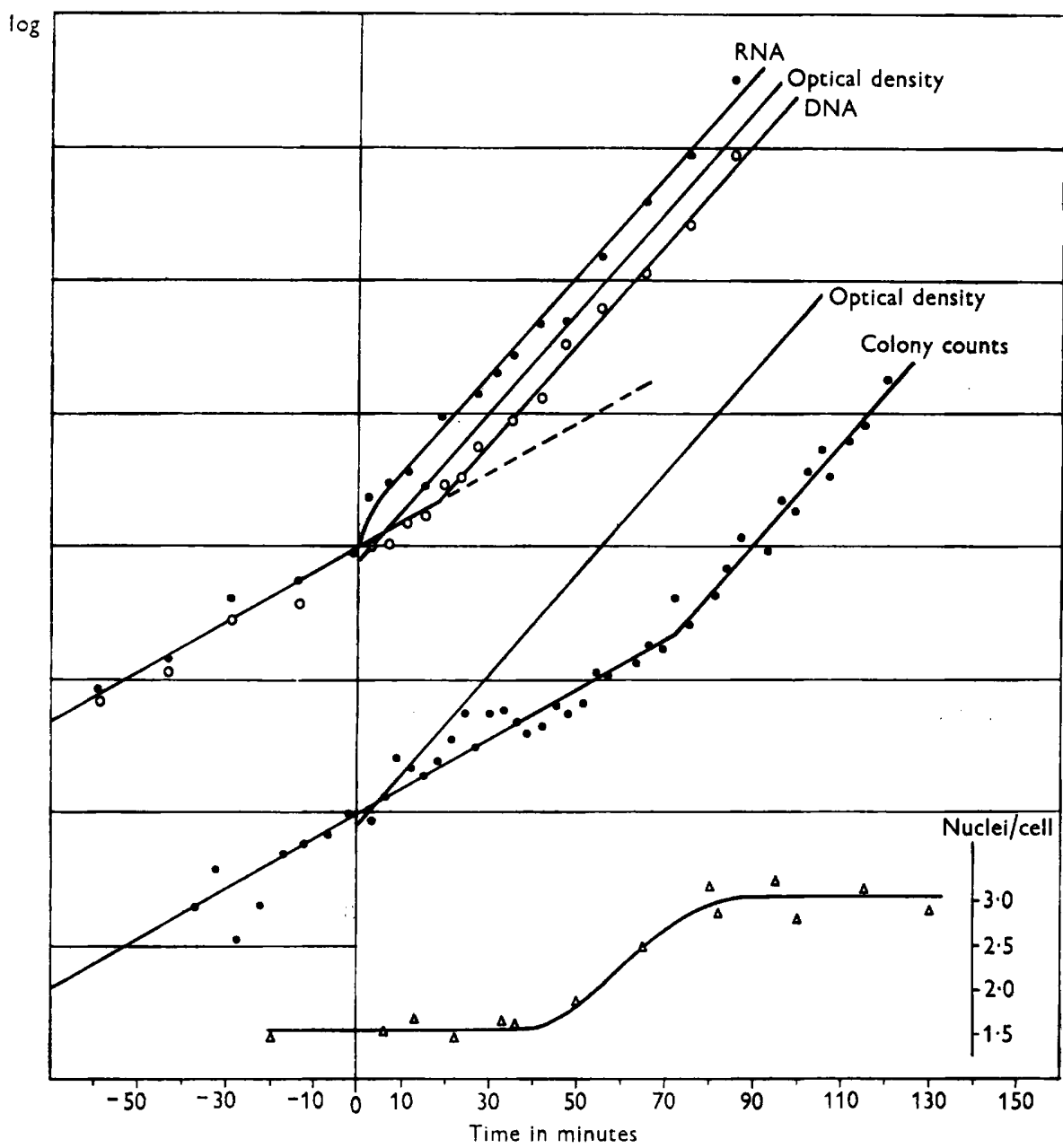

Fig. 1. Salmonella typhimurium culture at $37^{\circ}$, shifted from glucose salt minimal medium to broth at time 0 . Optical density, viable counts, RNA and DNA content were frequently determined. For the sake of clarity two separate sets of curves are presented: on top, optical density, RNA and DNA, and below, optical density and viable counts. In all cases the logarithms of the measured values are plotted against time and all values are transposed so as to make the curves representing balanced growth in minimal medium coincide. The distance between horizontal lines corresponds to one doubling. In lower right corner the average number of nuclei/cell obtained from direct counts on stained preparations are plotted against time. 
between a succinate salt medium and broth; in this case the final growth rate in broth (average generation time: $22 \mathrm{~min}$.) was more than three times the pre-shift rate (average generation time: $69 \mathrm{~min}$.) but, again, an abrupt change from the low to the high rate of cell division occurred about $70 \mathrm{~min}$. after the shift.

Several shifts up were performed with Salmonella typhimurium between different pairs of media. Media with amino acids, casein hydrolysate or the more complete defined medium 199 were substituted for broth in shifts from the usual glucose-salt medium; in other experiments, pre-shift growth rates as low as $\mathbf{0 . 6}$ doublings/hr. were obtained by using single amino acids or rhamnose as carbon and energy sources instead of glucose. In all cases the transition pattern of Fig. 1 was observed and the changes in the rates of DNA synthesis and cell division always occurred about 20 and $70 \mathrm{~min}$., respectively, after the shift. Thus, at a given temperature, the time during which the low, preshift rates were maintained was independent of the absolute growth rates in the media between which the shift was made.

Experiments done at lower temperatures with Salmonella typhimurium showed the usual dissociation pattern, but with a change in the time scale. Shifts were carried out at $30^{\circ}$ and $25^{\circ}$ between the glucose salt medium and broth; the results are presented in Table 1 together with data from a $37^{\circ}$ experiment.

Table 1. Shifts of Salmonella typhimurium from glucose salts medium to broth at different temperatures

\begin{tabular}{|c|c|c|c|c|c|c|}
\hline \multirow[b]{3}{*}{ Temp. } & $\mathrm{Cn}_{2}$ & h & \multicolumn{2}{|c|}{$\begin{array}{l}\text { Time of change (min.) } \\
\text { in rate of }\end{array}$} & \multicolumn{2}{|c|}{ Factor of increase in } \\
\hline & & & DNA & Cell & DNA/ & Mass/ \\
\hline & Pre-shift & Final & synthesis & division & cell & cell \\
\hline $37^{\circ}$ & $1 \cdot 20$ & $2 \cdot 40$ & 25 & 75 & $\mathbf{2 \cdot 0}$ & $2 \cdot 6$ \\
\hline $30^{\circ}$ & 0.73 & $1 \cdot 75$ & - & 90 & - & 2.5 \\
\hline $25^{\circ}$ & 0.62 & 1.15 & 60 & 150 & 1.8 & $2 \cdot 3$ \\
\hline
\end{tabular}

In the preceding paper we demonstrated that, during balanced growth, the size and composition of Salmonella typhimurium cells depend almost entirely on the medium and not on temperature. In all the media tested the growth rate at $37^{\circ}$ was about twice that obtained at $25^{\circ}$. The figures of Table 1 show that, like the generation time, the periods during which the pre-shift rates of DNA synthesis and of cell division are maintained are roughly doubled when the temperature is reduced from $37^{\circ}$ to $25^{\circ}$. In fact, our experiments indicate that the set of curves of Fig. 1 can be used to illustrate shift experiments carried out at different temperatures simply by adjusting the time scale. For instance, $1 \mathrm{hr}$. at $37^{\circ}$ is equivalent, in all respects, to about $2 \mathrm{hr}$. at $25^{\circ}$.

In a shift down the dissociation pattern differs in several ways from that of a shift up. Fig. 2 shows a typical experiment in which cells from a broth culture of Salmonella typhimurium were collected by filtration, washed on the filter and resuspended in glucose salt medium. Immediately after transfer, net synthesis of mass and RNA stopped for 30-40 min. (indeed, the amount of 
RNA usually dropped slightly). During this period, cell division and DNA synthesis continued until the cell number had increased by about a factor of two and the amount of DNA somewhat less. As a result the average number of nuclei/cell was reduced; in two experiments it decreased from $2 \cdot 4$ to 1.6 and from 2.5 to $1 \cdot 7$, respectively. The dissociation of the synthetic activities observed during the first 30-40 min. after the shift down achieved a partial reversion to the size and composition characteristic of cells growing in minimal

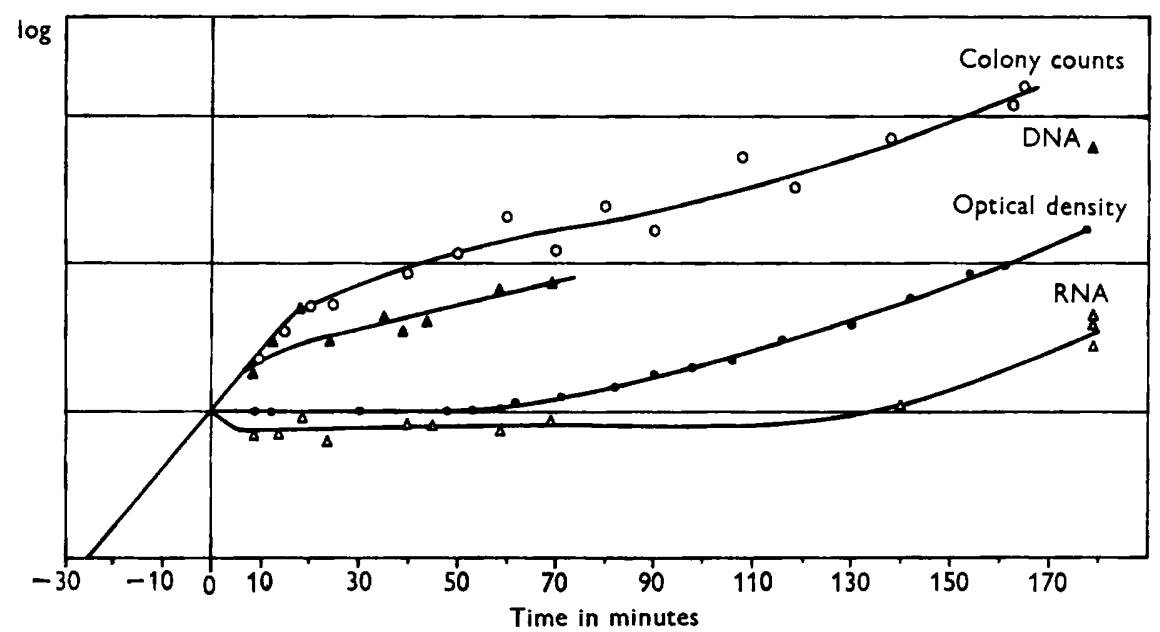

Fig. 2. Salmonella typhimurium culture at $37^{\circ}$ shifted from broth to glucose salt minimal medium at time 0 . Optical density, viable counts, RNA and DNA content were frequently determined. The logarithms of the measured values are plotted against time and all values are transposed so as to make the curves representing balanced growth in broth coincide. The distance between horizontal lines corresponds to one doubling.

medium. Complete reversion, however, took several hours, during which time the rates of synthesis of RNA and of cell mass gradually increased until the minimal medium values were reached.

Parallel platings on broth and on minimal agar gave identical colony counts; this showed that the lag in RNA and mass increase following a shift down was not, even in part, caused by loss of viability.

Several authors have shown that addition to a minimal medium culture of, for example, an amino acid, results in repression of the production of enzymes concerned with the synthesis of this compound (see, for example, Vogel, 1957). It may be assumed that, during growth in broth, a large number of enzymes are repressed. The kinetics of this 'collective repression' of enzyme synthesis was studied by carrying out first a shift up (from glucose minimal medium to broth) and then, at various times thereafter, a shift doron (back to glucose minimal medium). The optical density was frequently measured in all the cultures and, after each shift doron, the culture was followed until the definitive rate of mass increase had been established. A plot of the logarithm of the optical densities versus time is presented in Fig. 3. The transfer of the original minimal medium culture to broth took place at time 0 and the steep 
line represents the growth of the broth culture. The times of return to minimal glucose medium are marked on this line by arrows. The figure shows that the first 10-15 min. in broth do not affect the ability of the cells to resume growth at optimal rate in minimal medium. Thereafter, an increasing lag is observed until, after about $150 \mathrm{~min}$. in broth (i.e. approximately a 64-fold increase in bacterial mass), it reaches a maximum value. All cultures retransferred to

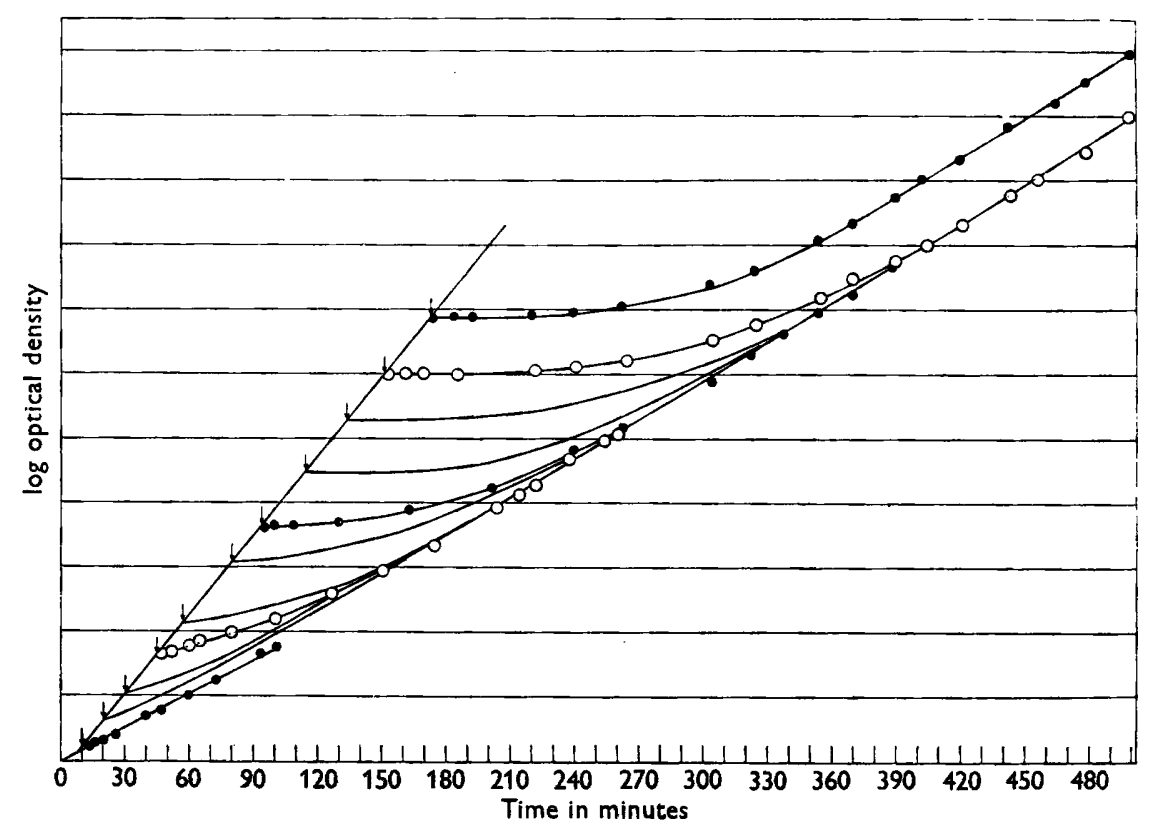

Fig. 3. A glucose salt minimal medium culture of Salmonella typhimurium at $37^{\circ}$ was diluted with broth at time 0 and the optical density of the culture followed. At various times after the shift samples of the culture were returned to glucose salt minimal medium and the optical density followed. The logarithms of the optical densities are plotted against time. The steep linear curve represents the growth curve of the broth culture and the arrows mark the time of return to minimal medium. The opticaldensity curves of the minimal medium cultures are transposed so as to obtain continuity with the broth curve at the time of transfer. For reason of clarity experimental points are not shown on all curves. The distance between horizontal lines corresponds to one doubling.

minimal medium between 15 and $150 \mathrm{~min}$. after the shift up ultimately follow the same growth curve. This indicates that during this time the length of the lag, or recovery period, is proportional to the time of growth in broth minus $15 \mathrm{~min}$.

Finally, shifts down were carried out between the complete glucose salt medium and the same medium without a nitrogen source. As in the experiments described above, cell division continued after the shift in the absence of net increase in cell mass, until the average cell size had been reduced to half. The cell size attained corresponds fairly well to the value obtained by extrapolating to zero growth rate in Fig. 1 of the preceding paper. No loss of viability seemed to occur during the first $3 \mathrm{hr}$. after the shift to minimal medium without 
nitrogen source. This was indicated by the fact that, upon addition of a nitrogen source or broth, the optical density of the culture immediately started increasing as in an ordinary shift up.

\section{DISCUSSION}

In the preceding paper (Schaechter et al. 1958) we showed that, under conditions of unrestricted balanced growth, mass, RNA and DNA/cell change exponentially with the growth rate. The transition from one state of balanced growth to another therefore requires a period of adjustment, the characteristies of which depend on the direction of the shift.

Shifts with rate increase (shift up) show a remarkably constant pattern under various experimental conditions. With different strains and at different temperatures it is uniformly found that the low, pre-shift rates of cell division and of DNA synthesis are maintained for well-defined periods, whereas the rates of mass increase and of RNA synthesis rapidly change to higher values (see Fig. 1 and Table 1).

This pattern is strikingly different from that observed when raising the temperature of a broth culture from $25^{\circ}$ to $37^{\circ}$ (Lark \& Maaløe, 1956). In this case the low, $\mathbf{2 5}^{\circ}$, rate of division was maintained for about $20 \mathrm{~min}$., but the amount of DNA/cell almost doubled during the first 5-10 min. after raising the temperature. This burst of synthesis was followed by a period with no net increase in DNA and a second, but smaller, burst of synthesis. In the temperature shift, which involves no overt change in the definitive size and composition of the cells, the rate of DNA synthesis thus cycles between extremely high and low values before the final $37^{\circ}$ rate is established.

On the basis of diffusion considerations Rashevsky (1948) suggested that division might be the direct consequence of cells reaching a critical size dependent on the physiological state. If this mechanism applied to Salmonella typhimurium, division would be expected to stop completely after a shift up, until the cells which were about to divide at the time of the shift had reached the critical size characteristic of the new medium. Fig. 1 shows that division does not stop even for a short time but continues at the pre-shift rate for a very considerable period. The mechanism responsible for this rate maintenance effect is unknown and no simple model can be suggested.

In the case of DNA synthesis the period preceding the rate change is only about $20 \mathrm{~min}$. and it is difficult to decide from individual experiments whether the pre-shift rate is strictly maintained. However, the cumulated evidence from many experiments shows that no lag is produced and that the rate of synthesis during this $20 \mathrm{~min}$. period is very close to the pre-shift rate. Like the synthesis of cell wall material (the general term 'wall material' is used here because we lack precise knowledge about the components of the cell wall and/or membrane, which are directly involved in the division process), the synthesis of DNA thus seems to be governed by a rate-controlling mechanism which, for a definite period, counteracts the metabolic factors responsible for the rapid increase in the rates of RNA and protein synthesis after a shift up. 


\section{$614 \quad$ N. O. Kjeldgaard, O. Maalфe and M. Schaechter}

The two control mechanisms have characteristic common features: First, at a given temperature, the periods during which the low, pre-shift rates are maintained are constant and independent of the absolute growth rates in the two media. These periods therefore bear no simple relation to either the preor the post-shift generation times. Secondly, if, between two temperatures the generation time doubles the control periods also double (Table 1). Thirdly, when these controls are eventually overcome, the new and higher rates are rapidly established, irrespective of the difference between pre- and post-shift rates. On the other hand, the two mechanisms are entirely separable since, after a temperature shift, the control of DNA synthesis breaks down at once, that of cell division only after about $20 \mathrm{~min}$. at $37^{\circ}$ (Lark \& Maaløe, 1956).
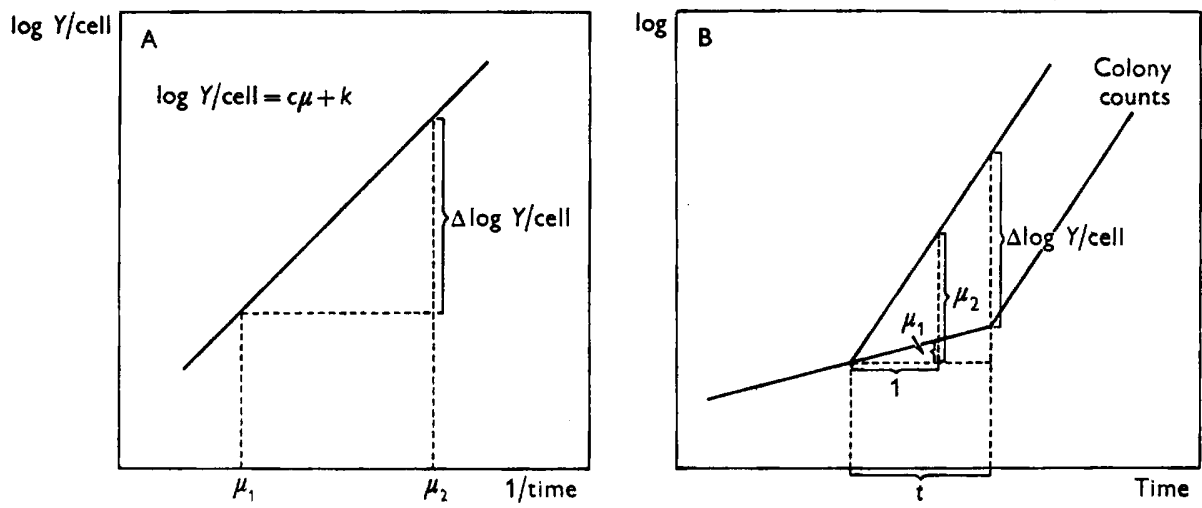

Fig. 4. Schematic illustration of the plot of cell content versus growth rate (A) (see preceding paper) and of a shift $u p$ experiment (B). $\mu_{1}$ and $\mu_{2}$ represent the rates before and after the shift, respectively $Y$ is the concentration of a given cell constituent. From the linear curve with slope $c$ in graph $A$ it is seen that the increase in the logarithm of $Y /$ cell $\left(\Delta \log Y /\right.$ cell) for a rate change from $\mu_{1}$ to $\mu_{2}$ equals $c\left(\mu_{2}-\mu_{1}\right)$. From graph $\mathbf{B}$, where $\mu_{1}$ and $\mu_{2}$ are the slopes of the growth curves and $t$ the time between rate changes, it follows that $\Delta \log Y /$ cell $=t\left(\mu_{1}-\mu_{2}\right)$. Consequently, for any $\mu_{1}$ and $\mu_{2}, t$ equals $c$ (see text).

In the intact cell, DNA synthesis presumably takes place in the "nuclear regions', and it is natural to imagine that the system responsible for the synthesis of wall material forms part of the cell wall itself or of the cell membrane. The two functions therefore seem to be separated physiologically as well as anatomically from the cytoplasm. Within the cytoplasm the small-particle fraction, which seems to contain most of the RNA (Schachman, Pardee \& Stanier, 1952), may constitute a third system, distinguishable by being particularly sensitive to changes in the medium. As shown in Fig. 1, RNA synthesis is unique in the sense that, in a shift $u p$, the initial rate exceeds the definitive one.

The strict time schedule for the rate changes which is suggested by our experiments can also be deduced from the exponential relations between growth rate and cell mass and DNA content previously established (see Fig. 4 and legend). Assuming that the transition pattern regularly observed in 
our experiments holds for shifts between any pair of media, theoretical times for DNA and colony count rate-changes were calculated from the slopes of the semi-logarithmic plots of cell mass and cell DNA versus growth rate (Figs. 1 and 3 in preceding paper). Taking into account that the optical-density curve has a lag of about $5 \mathrm{~min}$., the theoretical times for changes in rate after the medium shift $\left(\right.$ at $37^{\circ}$ ) are: $18 \mathrm{~min}$. for DNA, and $66 \mathrm{~min}$. for colony counts. Both values are in good agreement with the experimental findings (about 20 and $70 \mathrm{~min}$.)

The classical representation of the growth curve begins with a lag phase and a period of increasing growth rate which leads into the phase of exponential growth. Often, an outgrown culture contains a large percentage of nonviable cells whose presence veils the true mass increase of the viable bacteria and creates an apparent lag in the optical-density curve. On the assumption that all viable cells in such a mixed population start growing at maximum rate, an estimate of the fraction of non-viable cells was obtained by extrapolating the linear part of the growth curve, as determined by optical-density measurements, to zero time. This estimate was found to be in close agreement with the results we obtained by comparing viable and total cell counts.

It therefore looks as if all viable bacteria after transfer to a new medium start almost immediately to increase in mass at maximum rate, as has already been suggested by Hershey (1938). Colony counts, on the other hand, remain constant for about $70 \mathrm{~min}$. (maintenance of zero rate), before changing suddenly to the maximal rate. From the results of similar experiments, published by Lark \& Maaløe (1956), a lag period for DNA synthesis of about $20 \mathrm{~min}$. can be estimated. The viable cells of an outgrown broth culture can be shown to possess a more complete spectrum of enzymic activities than the exponentially growing 'broth cells'. Thus, when shifts down (to glucose salt medium) were carried out at various times during the transition from the exponential to the 'resting' phase of a broth culture, we observed that the lag in mass synthesis normally following such a shift became progressively shorter as the broth culture approached the resting stage. During the decline phase of a broth culture, the cells therefore seem gradually to regenerate their full synthetic capacity. To support this point we have measured the $\beta$-galactosidase in brothgrowing cells of a constitutive strain of Escherichia coli (ML 308). It was found that during the exponential phase enzyme synthesis was repressed and that, as the culture passed into the decline phase repression was released, with the result that the amount of enzyme/cell increased considerably.

The experiment of Fig. 3 is compatible with the assumption that, after a shift up, the overall activity of the repressed enzymes is lost by dilution. This has already been shown to be the case for a number of inducible enzymes (see review by Cohn, 1957).

The dependency of cell size and overall chemical composition on the growth rate described by Schaechter et al. (1958), and the transition pattern discussed above lead to a few general conclusions and invite much speculation.

The results of the shift experiments indicate that two of the major synthetic functions of the bacterial cell form separate systems within which different, distinct control mechanisms operate. Thus, on the background of cytoplasmic 


\section{N. O. Kjeldgaard, O. Maalфe and M. Schaechter}

protein and RNA synthesis, the rates of which almost immediately respond to changes of medium or temperature, the synthesis of DNA and of cell wall material stand out as individual, autonomous functions.

It may be long before the mechanisms invoked to account for the transition pattern can be discussed in specific chemical terms. Speculations in this direction would seem premature. We want to stress again, however, that control mechanisms with the properties described above would suffice to account quantitatively for the observation that cell size and composition are determined uniquely by the growth rate afforded by the medium.

The evolutionary significance of our observations is difficult, if not impossible to assess. Superficially, it seems a paradox that the fewer the synthetic activities required during growth the bigger the cell. On the other hand, if the hypothesis advanced by Schaechter et al.(1958) is correct, the growth rate cannot increase upon transfer to a richer medium unless the protein synthesizing system first expands. In other words, the cell must grow bigger, and increase its RNA/nucleus ratio, in order to grow faster; without this adjustment growth in the richer medium would be unbalanced. In Salmonella typhimurium the necessary enrichment in RNA is effected through the rate control, which delays DNA synthesis by maintaining the old rate for about 20 min. after a shift up. In a sense, it may thus be said that the control mechanism enables the cells eventually to grow faster in the new medium, and thereby confers a selective advantage upon them. It should be borne in mind, however, that the control mechanism might conceivably be an integral and indispensable part of the DNA synthesizing system; if so, it makes no sense to talk about selective advantage in this connexion.

One of the authors (N.O.K.) was aided by grants from the Danish National Science Foundation and the Lilly Research Foundation and one (M.S.) by a postdoctoral fellowship grant from the American Cancer Society.

\section{REFERENCES}

CoHN, M. (1957). Contribution of studies on the $\beta$-galactosidase of Escherichia coli to our understanding of enzyme synthesis. Bact. Rev. 21, 140.

Hershey, A. D. (1938). Factors limiting bacterial growth. II. Growth without lag in Bacterium coli cultures. Proc. Soc. exp. Biol., N.Y. 38, 127.

LaRK, K. G. \& MaAløe, O. (1956). Nucleic acid synthesis and the division cycle of Salmonella typhimurium. Biochim. biophys. Acta, 21, 448.

Rashevsky, N. (1948). Mathematical Biophysics. Chicago: The University of Chicago Press.

Schachman, H. K., Pardee, A. B. \& Stanier, R. Y. (1952). Studies on the macromolecular organization of microbial cells. Arch. Biochem. Biophys. 38, 245.

Schaechter, M., MaAløe, O. \& KJeldgaARd, N. O. (1959). Dependency on medium and temperature of cell size and chemical composition during balanced growth of Salmonella typhimurium. J. gen. Microbiol. 19, 592.

Voget, H. J. (1957). Repression and induction as control mechanisms of enzyme biogenesis: the 'adaptive' formation of acetylornithase. In Chemical Basis of Heredity, p. 276. Baltimore: The Johns Hopkins Press. 